\title{
A Numerical Study of a Rotor Induced Flow Based on a Finite-State Dynamic Wake Model
}

\author{
L. G. A. FERREIRA ${ }^{1 *}$, C. C. PAGANI JÚNIOR ${ }^{2}$, \\ E. M. GENNARO ${ }^{3}$ and C. DE MARQUI JUNIOR ${ }^{4}$
}

Received on January 15, 2020 / Accepted on January 27, 2021

\begin{abstract}
A Helicopter rotor undergoes unsteady aerodynamic loads ruled by the aeroelastic coupling between the elastic blades and the dynamic wake induced by rotary wings. Modeling the dynamic interaction between the structural and aerodynamic fields is a key point to understand aeroelastic phenomena associated with rotor stability, flow induced vibration and noise generation, among others. In this study, we address the Generalized Dynamic Wake Model, which describes the inflow velocity field at the rotor disk as a superposition of a finite number of induced flow states. It is a mature model that has been validated based on experimental data and numerically investigated from an eigenvalue problem formulation, whose eigenvalues and eigenvectors provide a deeper insight on the dynamic wake behavior. The paper extends the results presented in the literature to date in order to support physical interpretation of inflow states drawn from the finite-state wake model for flight conditions varying from hover to edgewise flight. The discussion of the wake model mathematical formulation is also oriented towards practical engineering applications to fill a gap in the literature.
\end{abstract}

Keywords: unsteady wake, eigen-analysis, inflow states.

\section{INTRODUCTION}

In the early 1980s, Dale Pitt and David Peters [10] developed a closed-form wake model to describe the rotor induced flow (the downwash in the rotor disk) from a matrix set of linear ordinary differential equations in the time domain. The Pitt-Peters model, as it is known, applies to an actuator disk (a circular region with zero thickness with lift vector normal to its surface)

\footnotetext{
*Corresponding author: Lucas Gustavo Alves Ferreira - E-mail: lucas_gustavo.5@ hotmail.com

${ }^{1}$ São Paulo State University (UNESP), Câmpus de São João da Boa Vista, São João da Boa Vista, SP, Brazil - E-mail: lucas_gustavo.5@hotmail.com https://orcid.org/0000-0002-3592-4524

${ }^{2}$ São Paulo State University (UNESP), Câmpus de São João da Boa Vista, São João da Boa Vista, SP, Brazil - E-mail: c.pagani@unesp.br https://orcid.org/0000-0001-8900-1939

${ }^{3}$ São Paulo State University (UNESP), Câmpus de São João da Boa Vista, São João da Boa Vista, SP, Brazil - E-mail: elmer.gennaro@unesp.br https://orcid.org/0000-0003-1074-396X

${ }^{4}$ University of São Paulo (USP), Department of Aeronautic Engineering, São Paulo, SP, Brazil - E-mail: demarqui@sc.usp.br https://orcid.org/0000-0003-4058-1885
} 
under the hypothesis of incompressible potential flow. Modern dynamic wake models largely used in the rotorcraft industry, such as free wake, prescribed wake, vortex particle and finitestate wake rely on vorticity transport equations for incompressible flow. The vorticity produced by the unsteady pressure load on the rotary blades trails within the wake at low speed relative to the blade tip. Therefore, the wake dynamics falls into a low Mach number regime and the compressibility hypothesis is hence applicable [3].

In the finite-state wake model, the unsteady pressure field on the blades and the rotor induced flow distribution are expressed in terms of series expansions of azimuthally periodic functions (Fourier Series) and radial shape functions (Legendre polynomials of first and second kinds) in an ellipsoidal coordinate system. In despite of being able of relating the transient rotor loads to the transient induced flow field, the Pitt-Peters model is a low-order model including only the zeroth and first harmonics azimuthally and one radial shape function associated with each harmonic. The induced flow states predicted by the Pitt-Peters model have physical meaning of uniform flow, a side-to-side gradient, and a fore-to-aft gradient, which is a crude description of the dynamic behavior of the induced wake due to the lack of higher-order harmonic terms.

Still in the 1980s, David Peters e Chengjian He [4, 8] developed a higher-order unsteady wake model known as Peters-He Generalized Dynamic Wake Model. In the Peters-He model, both pressure and induced flow distribution were extended to include higher order harmonics associated with an arbitrary number of radial shape functions. The model is able of predicting inflow states associated with the normal component of the flow velocity induced by pressure discontinuities through the rotor disk. Since a finite number of harmonics and associated radial shape functions must be selected for numerical application, this is classified as a finite-state wake model.

The aerodynamic load acting on rotary blades at steady hover flight condition is obtained from the lifiting line theory in which the circulatory lift is influenced by the inflow velocity derived from the wake model. The pressure load at the rotor disk is discretized to account for the unsteady wake of a finite-bladed rotor, which renders the aerodynamic environment suitable for aeroelastic analysis. In addition, the Peter's finite-state generalized wake model represents the pressure load in terms of a set of time-domain diffential equations, which is useful for calculating modal damping in subcritical flight conditions and state-space modeling for active control of flutter and design of controllers [5].

The Peters-He model has been validated based on wind-tunnel experimental data and widely applied to rotor stability, vibration, control, aeroelasticity and handling quality applications [6]. The work of Shang [11] shows the Generalized Dynamic Wake Model can be numerically integrated with a non-linear rotary wing finite-element model to provide a compact, accurate and computationally efficient aeroelastic model. For a consistent coupling between finite-element and finite-state dynamic wake models, the radial shape functions should be properly selected to approximate the induced flow mode shapes to the vibration modes relevant to the structural analysis. The numerical convergence of the finite-state wake model was investigated by He [4], which proposed a useful criterion, named the table method, to set the radial shape functions and 
hence determining the number of inflow states associated with a set of selected harmonic modes. Therefore, for achieving high-fidelity aeroelastic modeling by using the finite-state wake model based on the table method, the inflow states should be selected accordingly.

Wang [12] recast the Generalized Dynamic Wake Model homogeneous equations to an eigenvalue problem and obtained the eigenvalues and eigenvectors corresponding to induced flow states within a range of flight conditions varying from hover to edgewise flight. A wake model eigen-analysis allows for the identification of inflow mode shapes associated with the induced flow eigenvectors. At hover or axial flight conditions, the induced flow eigenvalues are real numbers, but at critical angles of skewed flow two real eigenvalues might couple one with other to produce a pair of complex-conjugate eigenvalues, for which real and imaginary parts represent the damping and oscillating frequency of the associated induced flow mode shape, respectively. Besides providing a deeper insight on the behavior of the dynamic wake, the eigen-analysis has been applied to study blade flapping dynamics [12] and flap-lag-torsion damping [1] of elastic blades coupled with unsteady aerodynamics.

Subsequent efforts were undertaken to achieve a wake model able of predicting all components of a potential velocity field throughout the flow field, that is, below and above the rotor disk. In the 1990s, a work by Wen-Ming Cao and David Peters [7] revealed the existence of a second set of inflow states, besides those associated with pressure discontinuities through the rotor disk. Further developments led to the Hsieh-Duffy model, which includes inflow states associated with mass-source and enables the determination of all components of the flow velocity above the rotor disk [9]. Fei Zhongyang [2] obtained a closed-form formulation for the non-potential velocity field below the rotor plane in terms of velocities (and their adjoints) above the rotor and applied eigen-analysis to the dynamic wake model including inflow states associated with mass sources to describe the inflow velocity distribution outside the rotor disk for an arbitrary wake skew angle.

In this paper we focus on the Generalized Dynamic Wake model and review the Wang's eigenanalysis for a set of inflow states. The mathematical features of the wake model are related to the physical meaning of the associated induced flow states.

\section{THE DYNAMIC WAKE MODEL FORMULATION}

The Generalized Dynamic Wake Model proposed by Peters and He [4, 8] is applied to calculate the inflow field induced by rotary blades at the rotor disk. The flight conditions here addressed vary from hover to edgewise flight.

The nondimensional induced flow velocity normal to the rotor disc, $\lambda(\bar{r}, \psi)$, is modeled as a series expansion of azimuthally periodic harmonic functions (Fourier Series) associated with radial shape functions, $\Psi_{n}^{m}(\bar{r})$, according to the following equation

$$
\lambda(\bar{r}, \psi)=\sum_{m, n}^{\infty} \Psi_{n}^{m}(\bar{r})\left[a_{n}^{m}(\bar{t}) \cos (\bar{r} \psi)+b_{n}^{m}(\bar{t}) \sin (\bar{r} \psi)\right],
$$


where $\psi, \bar{r}$ and $\bar{t}$ denote azimuth angle, radial position normalized by the blade length and nondimensional time, $\Omega t$, respectively, where $\Omega$ is the rotor rotational speed ( $\mathrm{rad} / \mathrm{s})$. Therefore, equation 2.1 yields the inflow velocity at normalized radial position $\bar{r}$ and azimuthal angle $\psi$ as a function of time, and can be applied to obtain the inflow distribution at the surface of the rotor disk.

The expansion coefficients of the induced velocity, $a_{n}^{m}(\bar{t})$ and $b_{n}^{m}(\bar{t})$, play the role of timedependent induced flow states in the unsteady Dynamic Wake Model. The indexes $m$ and $n$, which represent the order of harmonic modes and the associated radial shape functions in the finite-state model, respectively, are determined based on the table method proposed by He [4].

The radial expansion functions $\Psi_{n}^{m}(\bar{r})$ span a complete space of linearly independent functions for each harmonic, being defined as

$$
\Psi_{n}^{m}(\bar{r})=\frac{1}{v} \bar{P}_{n}^{m}(v)
$$

in which $\bar{P}_{n}^{m}(v)$ is a normalized Legendre function of the first kind, and $v$ is an ellipsoidal coordinate defined as $v=\sqrt{1-\bar{r}^{2}},(-1 \leq v \leq 1)$. Such a normalized radial expansion improves the induced velocity convergence near the blade tip. Based on equation 2.2, the function $\Psi_{n}^{m}(\bar{r})$ is expanded as

$$
\Psi_{n}^{m}(\bar{r})=\sqrt{(2 n+1) H_{n}^{m}} \sum_{q=m, m+2, \ldots}^{n-1} \bar{r}^{q} \frac{(-1)^{\frac{q-m}{2}}(n+q) ! !}{(q-m) ! !(q+m) ! !(n-q-1) ! !},
$$

where

$$
H_{n}^{m}=\frac{(n+m-1) ! !(n-m-1) ! !}{(n+m) ! !(n-m) ! !} .
$$

The time-dependent induced inflow states are determined from the generalized dynamic wake homogeneous equations in a non-rotating reference system [4], which allows for decoupling the harmonic terms.

$$
\begin{gathered}
{\left[\begin{array}{ccc}
\ddots & & \\
& {\left[K_{n}^{m}\right]} & \\
& & \ddots
\end{array}\right]\left[\begin{array}{lll}
\ddots & & \\
& {\left[A_{j n}^{r}\right]} & \\
& & \ddots
\end{array}\right] \frac{d}{d t}\left\{\begin{array}{c}
\vdots \\
\left\{a_{j}^{r}\right\} \\
\vdots
\end{array}\right\}+} \\
+V_{\infty}\left[\begin{array}{ccc}
\ldots & {\left[\bar{L}_{j n}^{r m}\right]^{c}} & \ldots \\
\vdots &
\end{array}\right]^{-1}\left[\begin{array}{lll}
\ddots & \\
& {\left[B_{j n}^{r}\right]} & \\
& & \ddots
\end{array}\right]\left\{\begin{array}{c}
\vdots \\
\left\{a_{j}^{r}\right\} \\
\vdots
\end{array}\right\}=\frac{1}{2}\left\{\begin{array}{c}
\vdots \\
\{0\} \\
\vdots
\end{array}\right\}
\end{gathered}
$$




$$
\begin{aligned}
& {\left[\begin{array}{lll}
\ddots & & \\
& {\left[K_{n}^{m}\right]} & \\
& & \ddots
\end{array}\right]\left[\begin{array}{lll}
\ddots & & \\
& {\left[A_{j n}^{r}\right]} & \\
& & \ddots
\end{array}\right] \frac{d}{d t}\left\{\begin{array}{c}
\vdots \\
\left\{b_{j}^{r}\right\} \\
\vdots
\end{array}\right\}+} \\
& +V_{\infty}\left[\begin{array}{ccc} 
& \vdots & \\
\cdots & {\left[\bar{L}_{j n}^{r m}\right]^{s}} & \cdots \\
\vdots &
\end{array}\right]^{-1}\left[\begin{array}{lll}
\ddots & & \\
& {\left[B_{j n}^{r}\right]} & \\
& & \ddots
\end{array}\right]\left\{\begin{array}{c}
\vdots \\
\left\{b_{j}^{r}\right\} \\
\vdots
\end{array}\right\}=\frac{1}{2}\left\{\begin{array}{c}
\vdots \\
\{0\} \\
\vdots
\end{array}\right\}
\end{aligned}
$$

In Eqs. 2.3 and 2.4, $\left\{a_{j}^{r}\right\}$ and $\left\{b_{j}^{r}\right\}$ are steady inflow states and their time derivatives, $\left\{\dot{a}_{j}^{r}\right\}$ and $\left\{\dot{b}_{j}^{r}\right\}$, unsteady inflow states induced by the movement of a finite number of blades. $m$ and $r$ are indexes for harmonic modes and $n$ and $j$ for the associated radial shape functions. The subscripts $c$ and $s$ denote cosine and sine terms in an azimuthally periodic expansion. $V_{\infty}$ is the normalized free-stream velocity and $K_{n}^{m}$ are elements of the apparent mass matrix, which is related to the kinetic energy of the air mass accelerated through the rotor disk

$$
K_{n}^{m}=\frac{2}{\pi} H_{n}^{m} .
$$

The apparent mass matrix is a diagonal matrix carrying no coupling between harmonic modes and radial shape functions. The wake influence coefficients matrices, $\left[L_{j n}^{r m}\right]^{c, s}$, are identity matrices at hover or axial flight, but become full matrices coupling harmonic modes for flight at skewed angles.

The use of the expansion function $\bar{P}_{n}^{m}(v) / v$ renders $\left[A_{j n}^{r}\right]$ an identity matrix and $B_{j n}^{r}$ a diagonal blocks matrix coupling the radial shape functions for each harmonic mode, which leads to a three-dimensional wake model [12]. See He [4] for explicit definitions of the matrices $\left[B_{j n}^{r}\right]$ and $\left[L_{j n}^{r m}\right]^{c, s}$. Eqs. 2.3 and 2.4 apply to general flight condition, that is, from hover to edgewise flight.

\section{DYNAMIC WAKE MODEL EIGENVALUE FORMULATION}

The Generalized Dynamic Wake Model is formulated in the time domain as a linear problem whose solution, in terms of eigenvalues and eigenvectors, determines the induced flow states [12]. To achieve the eigen-problem formulation, Eqs. 2.3 and 2.4 are rewritten as follows for states associated with the cosine term:

$$
\left[K_{n}^{m}\right]\left[A_{j n}^{r}\right] \frac{d}{d t}\left\{a_{j}^{r}\right\}+V_{\infty}\left[L_{j n}^{r m c}\right]^{-1}\left[B_{j n}^{r}\right]\left\{a_{j}^{r}\right\}=\{0\}
$$

and sine term of the induced flow expansion:

$$
\left[K_{n}^{m}\right]\left[A_{j n}^{r}\right] \frac{d}{d t}\left\{b_{j}^{r}\right\}+V_{\infty}\left[L_{j n}^{r m s}\right]^{-1}\left[B_{j n}^{r}\right]\left\{b_{j}^{r}\right\}=\{0\} .
$$


The matrix $\left[B_{j n}^{r}\right]$ approaches $\left[\bar{A}_{j n}^{r}\right]^{-1}$, where, following He's notation [4], the overline symbol is used to define the latter matrix expansion in terms of the normalized Legendre function, $\bar{P}_{n}^{m}(v)$, as follows:

$$
\left[\bar{A}_{j n}^{r}\right]=\int_{0}^{1} \bar{P}_{j}^{m}(v) \bar{P}_{n}^{m}(v) v d v .
$$

The approximation holds strictly true in the limit of an infinite number of induced flow states. However, it is verified to be a good approximation even for a few-states wake model [8]. Using this approximation, the eigenvalue equation (for cosine states) writes

$$
\left[K_{n}^{m}\right] \frac{d}{d t}\left\{a_{j}^{r}\right\}+V_{\infty}\left[L_{j n}^{r m c}\right]^{-1}\left[\bar{A}_{j n}^{r}\right]^{-1}\left\{a_{j}^{r}\right\}=\{0\} .
$$

Using the identity $[L]^{-1}[A]^{-1}=[A L]^{-1}$, we have

$$
\left[K_{n}^{m}\right] \frac{d}{d t}\left\{a_{j}^{r}\right\}+V_{\infty}\left[\bar{A}_{n j}^{r} L_{j n}^{r m c}\right]^{-1}\left\{a_{j}^{r}\right\}=0,
$$

whose solution yields the induced inflow states in terms of the Generalized Dynamic Wake Model eigenvalues and eigenvectors. Therefore, the following solution is assumed for the $i^{\text {th }}$ cosine state

$$
\left\{a_{i}\right\}=\left\{\phi_{i}\right\} e^{\zeta_{i} V_{\infty} \bar{t}}
$$

where $\zeta_{i}$ is the $i^{t h}$ eigenvalue and $\phi_{i}$ is the associated eigenvector. Inserting equation 3.2 into 3.1 leads to

$$
\{\phi\} V_{\infty} e^{\zeta V_{\infty} \bar{t}}\left(\left[K_{n}^{m}\right] \zeta+\left[\bar{A}_{n j}^{r} L_{j n}^{r m c}\right]^{-1}\right)=0,
$$

for which a solution is given by

$$
\left|\left[K_{n}^{m}\right] \zeta+\left[\bar{A}_{n j}^{r} L_{j n}^{r m c}\right]^{-1}\right|=0 .
$$

Pre-multiplying the above equation by $\left[K_{n}^{m}\right]^{-1}$ yields

$$
|[D]+[I] \zeta|=0,
$$

where $[D]$ is the Dynamic Matrix

$$
[D]=\left[K_{n}^{m}\right]^{-1}\left[\bar{A}_{n j}^{r} L_{j n}^{r m c}\right]^{-1} .
$$

Following Wang [12], a change of variable is used to normalized the Dynamic Matrix, which leads to

$$
[D]=\left[K_{n}^{m}\right]^{-1 / 2}\left[\bar{A}_{n j}^{r} L_{j n}^{r m c}\right]^{-1}\left[K_{n}^{m}\right]^{-1 / 2}
$$

From such an eigenvalue normalization, the corresponding normalized eigenvectors are given by

$$
\{\bar{\phi}\}=\left[k_{n}^{m}\right]^{-1 / 2}\{\phi\}
$$


For numerics, a closed-form for matrix $\left[\bar{A}_{n j}^{r} L_{j n}^{r m c}\right]$, here denoted by $\tilde{L}$, was deduced by He [4] to account for the skew angle influence on the induced inflow states

$$
\tilde{L}=\left[A_{j n}^{r} L_{j n}^{r m c}\right]_{\text {closed-form }} .
$$

See also Wang [12] for more details and for a definition of a closed-form matrix for the inverse of the matrix $\tilde{L}$. For cosine states

$$
\begin{gathered}
{\left[\tilde{L}_{j n}^{0 m}\right]^{c}=X^{m}\left[\Gamma_{j n}^{0 m}\right]} \\
{\left[\tilde{L}_{j n}^{r m}\right]^{c}=\left[X^{|m-r|}+(-1)^{l} X^{|m+r|}\right]\left[\Gamma_{j n}^{r m}\right],}
\end{gathered}
$$

and for sine states

$$
\left[\tilde{L}_{j n}^{r m}\right]^{s}=\left[X^{|m-r|}-(-1)^{l} X^{|m+r|}\right]\left[\Gamma_{j n}^{r m}\right]
$$

with $l=\min (r, m), X=\tan \left(\left|\frac{\chi}{2}\right|\right)$ and $\chi=\frac{\pi}{2}-\alpha$, where $\alpha$ is the disk angle of attack, according to Figure 1 (notice that $0 \leq X \leq 1$ ).

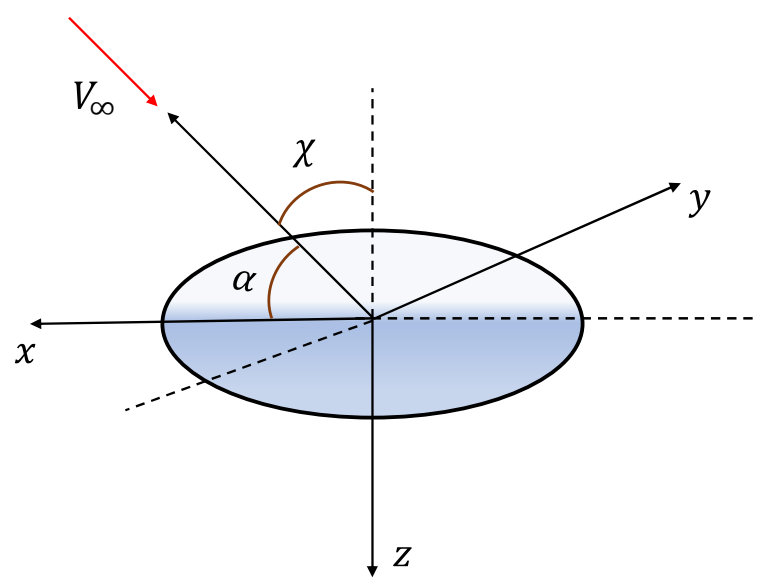

Figure 1: View from above the rotor disk with the free stream from left to right for skewed incoming flow.

The function $\Gamma_{j n}^{r m}$ is defined according to

- For $r+m$ even

$$
\Gamma_{j n}^{r m}=\frac{(-1)^{\frac{n+j-2 r}{2}}}{\sqrt{H_{n}^{m} H_{j}^{r}}} \frac{2 \sqrt{(2 n+1)(2 j+1)}}{(j+n)(j+n+2)\left[\left((j-n)^{2}-1\right)\right]}
$$


- For $r+m$ odd and $j=n \pm 1$

$$
\Gamma_{j n}^{r m}=\frac{\pi}{2 \sqrt{H_{n}^{m} H_{j}^{r}}} \frac{\operatorname{sgn}(r-m)}{\sqrt{(2 n+1)(2 j+1)}}
$$

- For $r+m$ odd and $j \neq n \pm 1$

$$
\Gamma_{j n}^{r m}=0
$$

\section{NUMERICAL RESULTS AND DISCUSSION}

The eigenvalue formulation for the Generalized Dynamic Wake Model was numerically solved for some combinations of harmonic and radial shape functions. The skew angle varies from $0^{\circ}$ (hover) to $90^{\circ}$ (edgewise flight), to account for its effect on the induced flow characteristics. Table 1 shows the harmonic $(m, r)$ indexes, the associated radial shape functions indexes $(n, j)$, selected based on the table method [4], and the respective number of inflow states for a wake model with highest harmonic number equals to $16(M=16)$, which accounts for 81 inflow states and is the highest order harmonic number wake model investigated in this paper.

Table 1: Harmonics and associated radial shape functions for $M=16$ based on the table method.

\begin{tabular}{ccc}
\hline$m, r$ & $n, j$ & Inflow States \\
\hline 0 & $1,3,5,7,9,11,13,15,17$ & 9 \\
\hline 1 & $2,4,6,8,10,12,14,16$ & 8 \\
\hline 2 & $3,5,7,9,11,13,15,17$ & 8 \\
\hline 3 & $4,6,8,10,12,14,16$ & 7 \\
\hline 4 & $5,7,9,11,13,15,17$ & 7 \\
\hline 5 & $6,8,10,12,14,16$ & 6 \\
\hline 6 & $7,9,11,13,15,17$ & 6 \\
\hline 7 & $8,10,12,14,16$ & 5 \\
\hline 8 & $9,11,13,15,17$ & 5 \\
\hline 9 & $10,12,14,16$ & 4 \\
\hline 10 & $11,13,15,17$ & 4 \\
\hline 11 & $12,14,16$ & 3 \\
\hline 12 & $13,15,17$ & 3 \\
\hline 13 & 14,16 & 2 \\
\hline 14 & 15,17 & 2 \\
\hline 15 & 16 & 1 \\
\hline 16 & 17 & 1 \\
\hline
\end{tabular}

Next, results are presented for eigenvalues and mode shapes corresponding to a set of selected inflow states. 


\subsection{Eigenvalue Analysis}

Figure 2 shows the eigenvalues for a wake model with a single harmonic, $m=0$, and associated radial shape functions given by $n=1,3,5,7,9$ (according to the table method, these are the radial shape functions associated with $m=0$ for a 8-harmonic wake model).

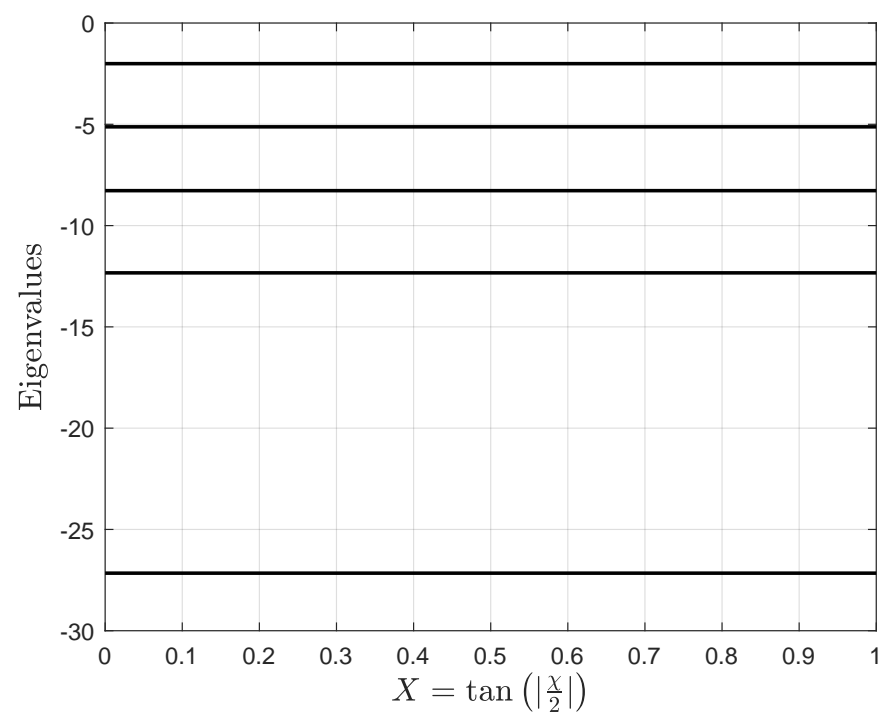

Figure 2: Inflow eigenvalues as a function of the wake skew angle for inflow states with $m=0$ and $n=1,3,5,7,9$.

According to figure 2, the wake model eigenvalues are constant real numbers throughout the range of skew angles. Since the model is simulated for $m=0$ only, there is no coupling between harmonic functions, which leads to real eigenvalues within the entire range of wake skew angles.

Figures 3 and 4 show the eigenvalues real and imaginary parts for a wake model with $M=2$ and radial shape functions selected based on the table method, which corresponds to 4 inflow states.

According to figure 3, two eigenvalues couple one with another at a skew angle function of around $0.3\left(\chi=33^{\circ}\right)$. The two real eigenvalues split into a pair of complex-conjugate eigenvalues whose imaginary parts, which arise at the skew angle the real parts coalesce each other, are shown in figure 4. The coupled inflow state has associated to it a damping (related to the eigenvalue real part) and an oscillation frequency (related to the eigenvalue imaginary part).

Figures 5 and 6 show the eigenvalues real and imaginary parts for a wake model with $M=4$ and radial shape functions selected based on the table method, which corresponds to 9 inflow states. There are now three coupling between real eigenvalues at different wake skew angles. 


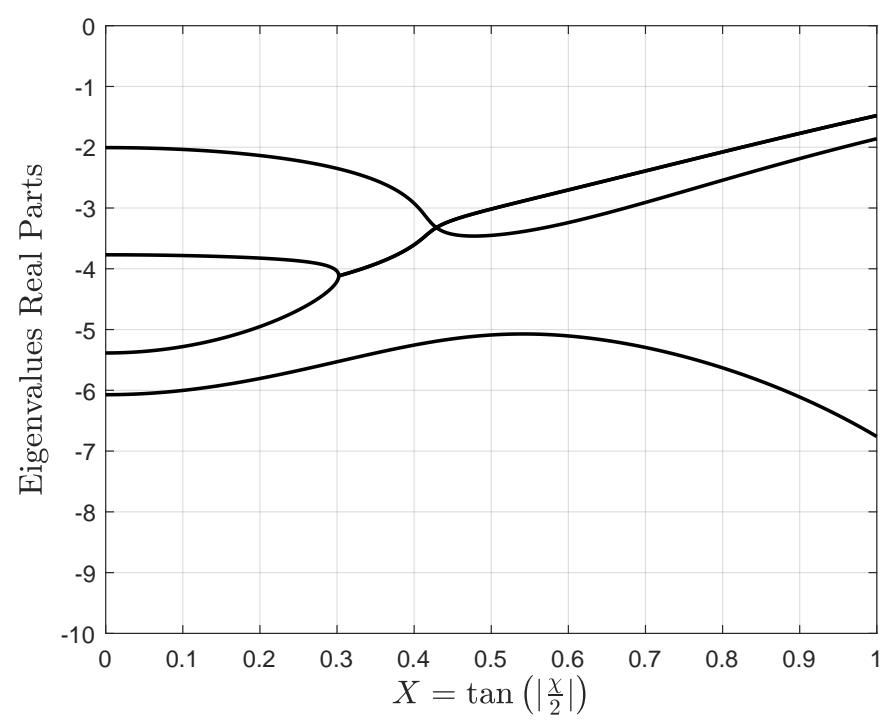

Figure 3: Inflow eigenvalues real parts as a function of the wake skew angle for inflow states with harmonics $m=0,1,2$ and radial shape functions based on the table method.

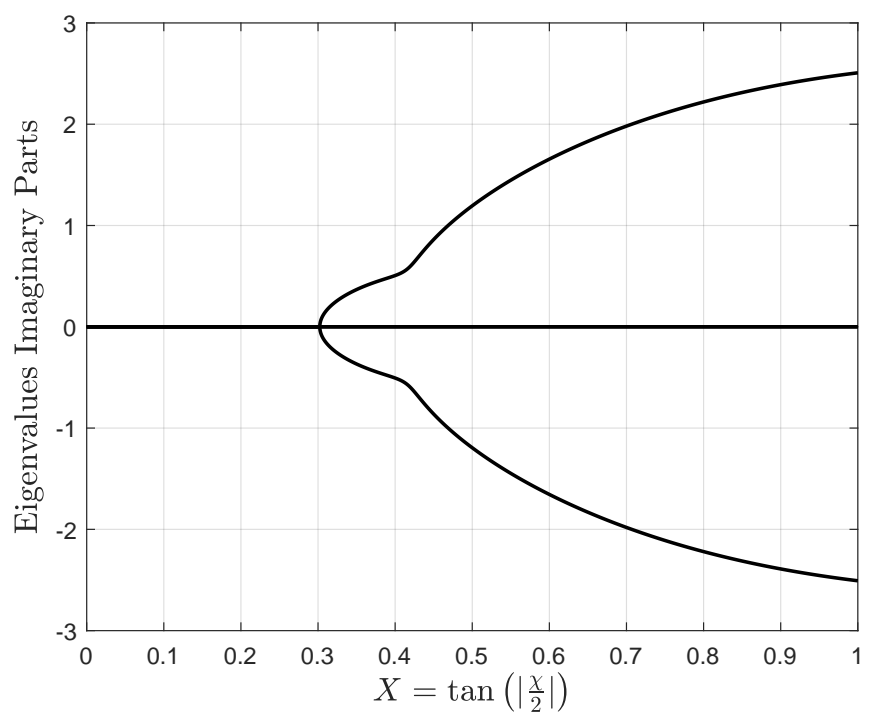

Figure 4: Inflow eigenvalues imaginary parts as a function of the wake skew angle for inflow states with harmonics $m=0,1,2$ and radial shape functions based on the table method. 


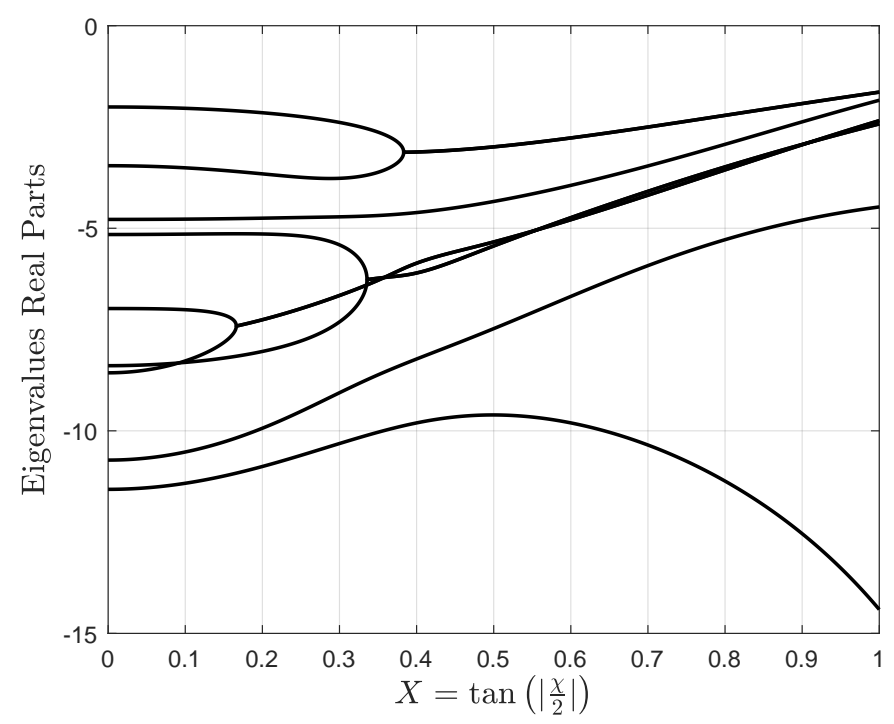

Figure 5: Inflow eigenvalues real parts as a function of the wake skew angle for inflow states with harmonics $m=0,1,2,3,4$ and radial shape functions based on the table method.

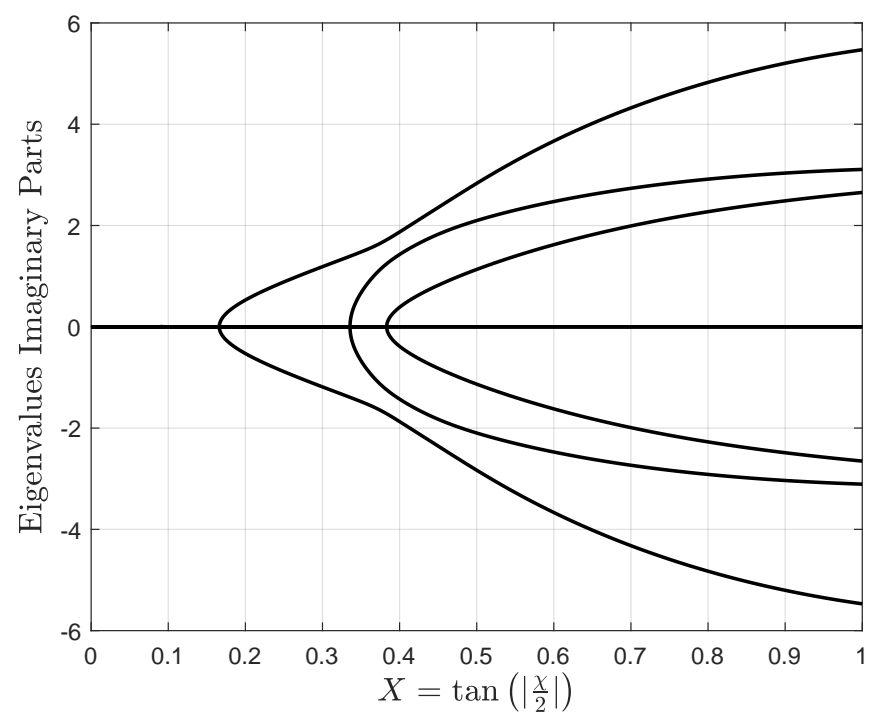

Figure 6: Inflow eigenvalues imaginary parts as a function of the wake skew angle for inflow states with harmonics $m=0,1,2,3,4$ and radial shape functions based on the table method. 
Figures 7 and 8 show the eigenvalues real parts for a wake model with $M=8$ and $M=16$, with radial shape functions selected based on the table method, which correspond to 25 and 81 inflow states, respectively. The eigenvalues distribution for $M=8$, as a function of the wake skew angle, is in accordance with results previously presented by Wang [12]. According to the results, the dynamic wake model predicts a strong coupling between inflow states as the wake skew angle increases.

\subsection{Induced Flow Mode Shapes}

According to Wang [12], the mode shapes associated with each selected inflow state are obtained from radial expansion functions and the induced flow eigenvectors based on the following equations for cosine states

$$
\lambda(\bar{r}, \psi)=\sum_{r, j}^{\infty} \Psi_{n}^{m}(\bar{r}) \bar{\phi}_{j}^{r} \cos (\bar{r} \psi)
$$

and sine states

$$
\lambda(\bar{r}, \psi)=\sum_{r, j}^{\infty} \Psi_{n}^{m}(\bar{r}) \bar{\phi}_{j}^{r} \sin (\bar{r} \psi) .
$$

In this study, equation 4.1 was applied to simulate mode shapes for wake models with maximal harmonic numbers given by $M=2$ and $M=16$, with radial shape functions for each harmonic selected based on the table method, which accounts for 4 inflow states for $M=2$ and 81 inflow states for $M=16$. Table 2 shows harmonics, radial shape functions and induced flow eigenvalues at hover for the four inflow states wake model, for reference. The inflow states eigenvalues are given in Fig. 3 (real parts) and Fig. 4 (imaginary parts) from hover to edgewise flight.

Table 2: Harmonics and associated radial shape functions for $M=2$ based on the table method.

\begin{tabular}{ccc}
\hline Harmonics $(m, r)$ & Radial Shape Functions $(n, j)$ & Eigenvalues at Hover \\
\hline 0 & 1 & -2.0062 \\
\hline 0 & 3 & -6.0722 \\
\hline 1 & 2 & -3.7699 \\
\hline 2 & 3 & -5.3856 \\
\hline
\end{tabular}

Figure 9 outlines the mode shapes and nodal lines (no inflow through the rotor disk along the line path) at hover $(X=0)$ for each inflow state shown in table 2. A mode shape stands for the inflow distribution at the rotor disk, that is, the induced velocity field normal to rotor plane within the rotor disk. According to figure 9, the mode shapes are azimuthally symmetric at hover flight. The shape function associated with the eigenvalue $\zeta=-2.0062$ corresponds to a fundamental inflow state, $(m, n=0,1)$, with no nodal line. Even for a low order wake model, it turns out the mode shape dependence on the harmonic and associated radial shape functions. 


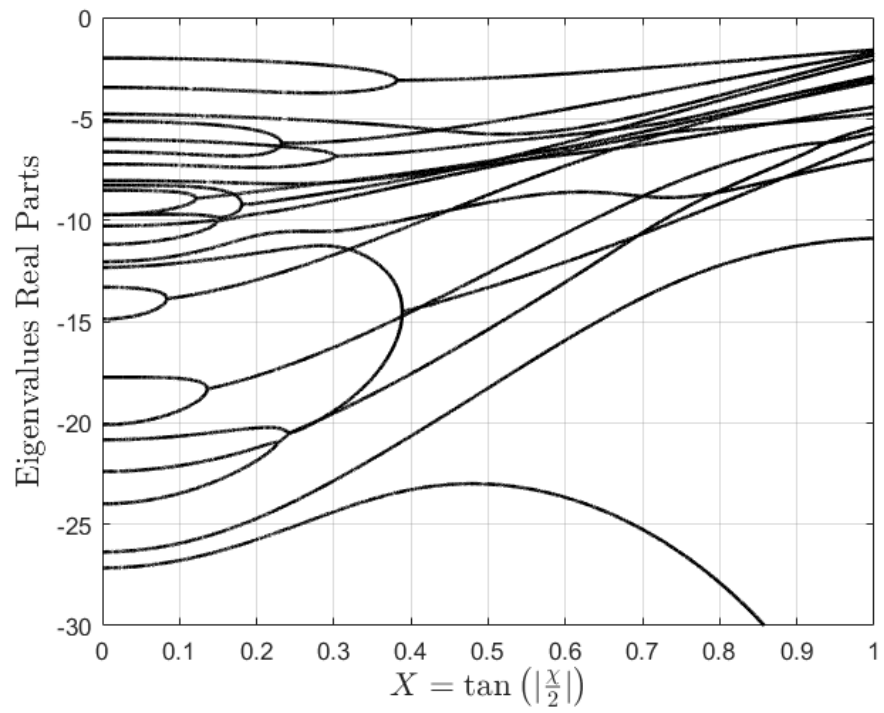

Figure 7: Inflow eigenvalues real parts as a function of the wake skew angle for inflow states with harmonics $m=0,1,2,3,4,5,6,7,8$ and radial shape functions based on the table method.

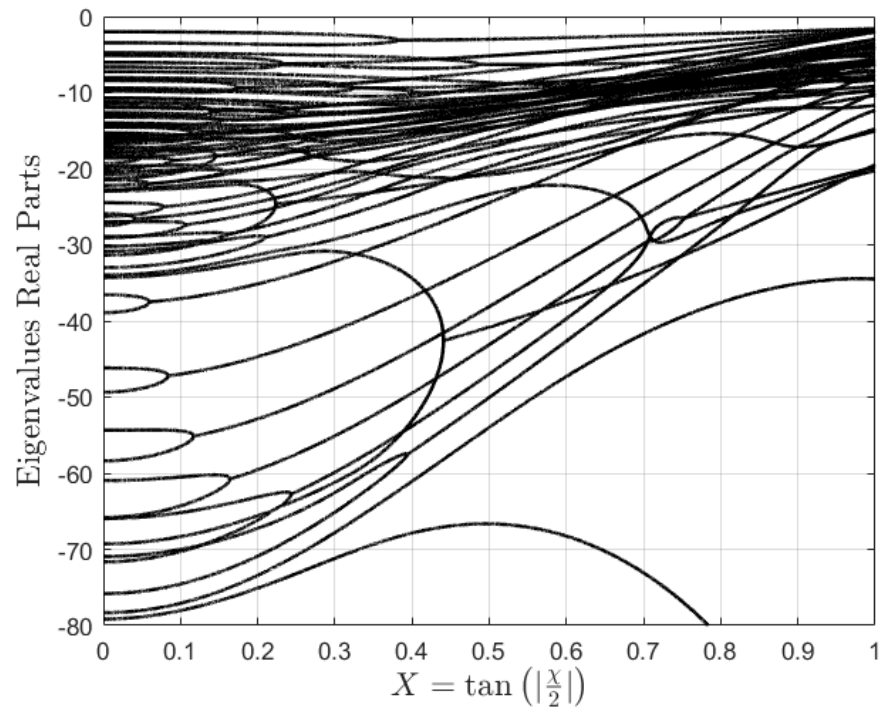

Figure 8: Inflow eigenvalues real parts as a function of the wake skew angle for inflow states with harmonics $m=0,1,2,3,4,5,6,7,8,9,10,11,12,13,14,15,16$ and radial shape functions based on the table method. 


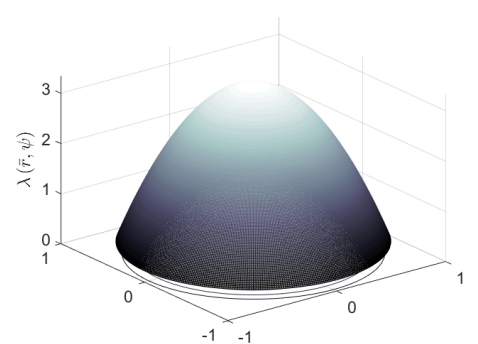

(a) Mode shape $(m, n=0,1), X=0$

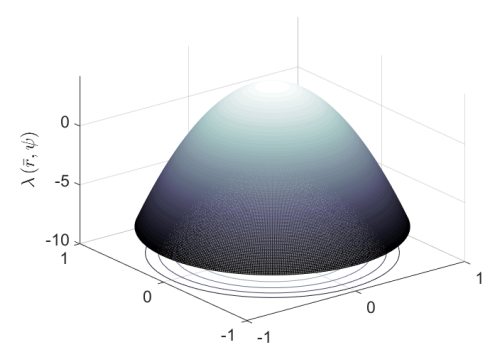

(c) Mode shape $(m, n=0,3), X=0$

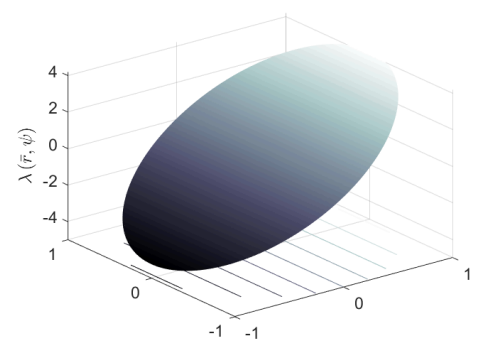

(e) Mode shape $(m, n=1,2), X=0$

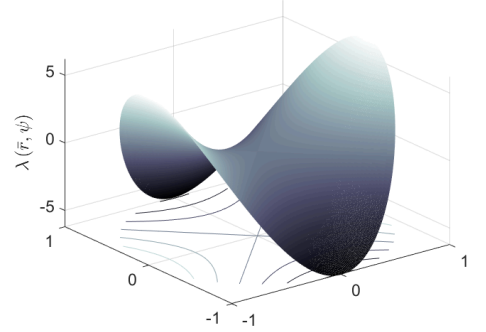

(g) Mode shape $(m, n=2,3), X=0$

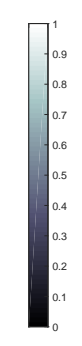

(b) Nodal line $(m, n=0,1), X=0$

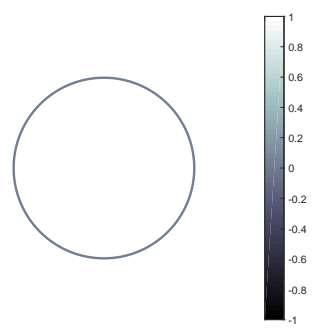

(d) Nodal line $(m, n=0,3), X=0$

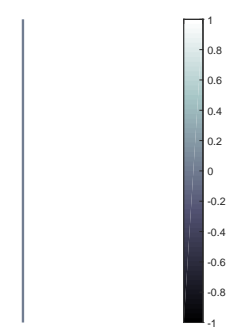

(f) Nodal line $(m, n=1,2), X=0$

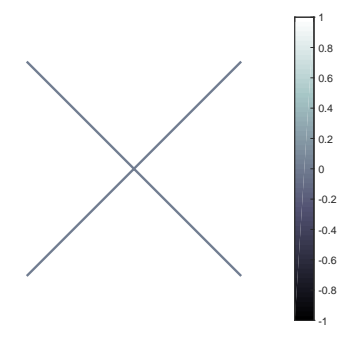

(h) Nodal line $(m, n=2,3), X=0$

Figure 9: Induced flow mode shapes and nodal lines for a 2-harmonic wake model at hover $(X=$ $0)$. 


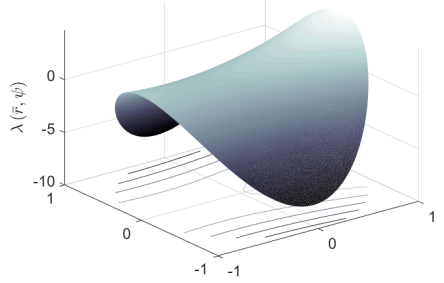

(a) Mode shape $(m, n=0,1), X=1$

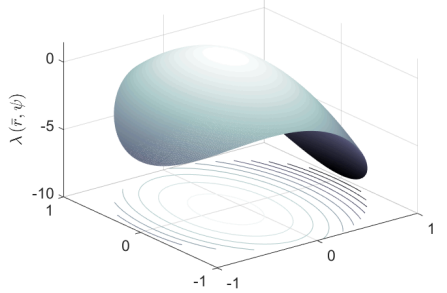

(c) Mode shape $(m, n=0,3), X=1$

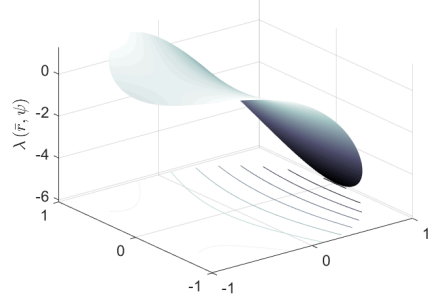

(e) Mode shape (coupled), $X=1$

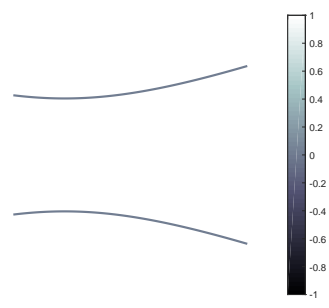

(b) Nodal line $(m, n=0,1), X=1$

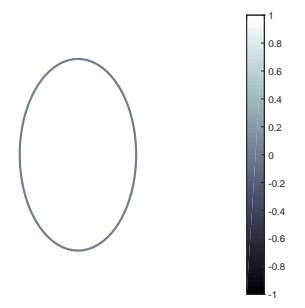

(d) Nodal line $(m, n=0,3), X=1$

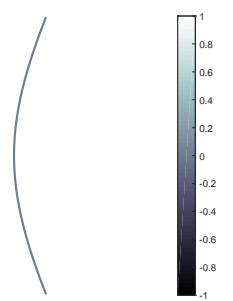

(f) Nodal line (coupled), $X=1$

Figure 10: Induced flow mode shapes and nodal lines for a 2-harmonic wake model at edgewise flight $(X=1)$.

Figure 10 shows the induced flow mode shapes at edgewise flow $X=1$. Overall, it turns out a strong influence from the flight condition on the mode shapes, which became asymmetric, as can be easily drawn from nodal lines path. At edgewise flight, the mode shapes associated with inflow states corresponding to $(m, n=1,2)$ and $(m, n=2,1)$ coupled one another to originate a new inflow state. The resultant inflow state mode shape bears the inflow distribution characteristics from both mode shapes contributing to the coupling. In some cases, there is a dominant inflow mode determining the shape of the resultant mode.

Figure 11 shows mode shapes and nodal lines for the inflow states of a 16-harmonic wake model, with associated radial shape functions selected based on the table method. The inflow states correspond to $(m, n=0,2)$ and $(m, n=16,17)$ at hover and edgewise flight conditions. For the 


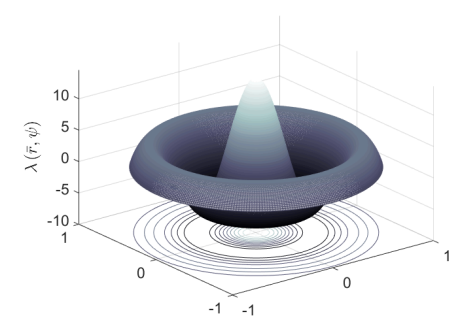

(a) Mode shape $(m, n=0,2), X=0$

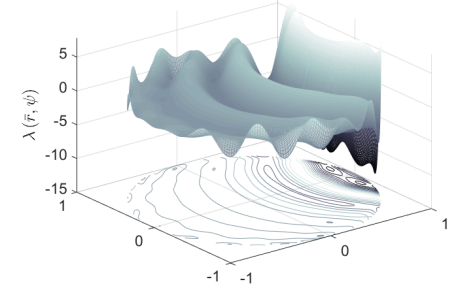

(c) Mode shape $(m, n=0,2), X=1$

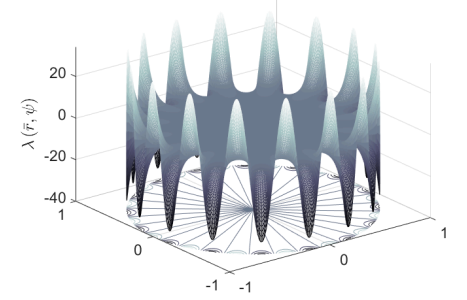

(e) Mode shape $(m, n=16,17), X=0$

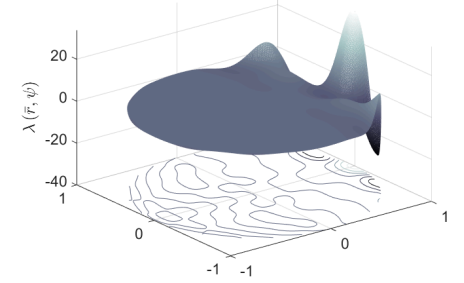

(g) Mode shape $(m, n=16,17), X=$ 1

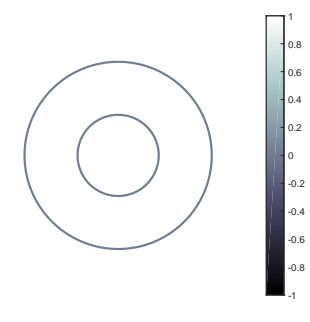

(b) Nodal line $(m, n=0,2), X=0$

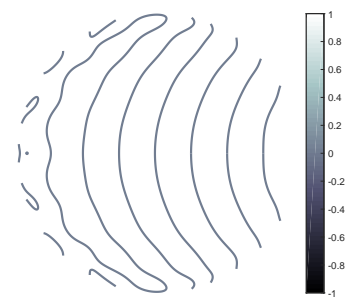

(d) Nodal line $(m, n=0,2), X=1$

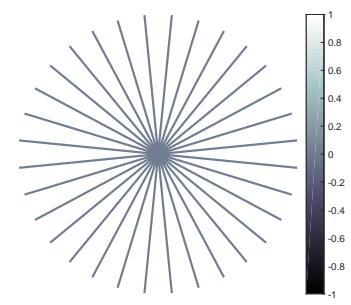

(f) Nodal line $(m, n=16,17), X=0$

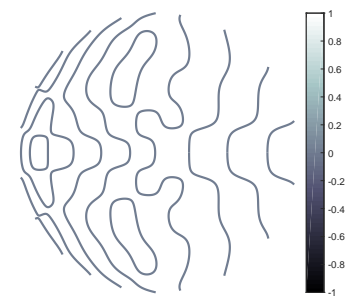

(h) Nodal line $(m, n=16,17), X=1$

Figure 11: Induced flow mode shapes and nodal lines for a 16-harmonic wake model at hover and edgewise flight. 
lower harmonic inflow state the induced flow velocity is higher at the center of the rotor, whereas for the highest harmonic number inflow state the inflow distribution is most uniform, but peaks around the rotor edge. For $m=0$, there is no radial node lines, but for $m=16$, there is also 16 radial node lines. Even for high order inflow states, the inflow distribution remains azimuthally symmetry at hover, which is clearly seen from the nodal line contours. However, for both the lower and higher harmonic order inflow states, the inflow distribution is strongly influenced by the wake skew angle.

\section{CONCLUSION}

This study addressed a finite state unsteady wake model based on an eigenvalue-problem formulation for the wake homogenous equations for a range of wake skew angles varying from hover to edgewise flight. The inflow states mode shapes were associated with the dynamic wake model eigenvectors. The eigenvalues are real numbers for a wake induced at axial flight, but are prone to couple one each other at critical wake skew angles, resulting in pairs of complex-conjugate eigenvalues whose real part relates to damping and imaginary parts to oscillating frequency of induced flow modes. The inflow state mode shapes show azimuthal symmetry at axial flight, but becomes strongly asymmetric as the inflow incidence angle increases, which generally leads to a new inflow distribution through the rotor disk.

\section{Aknowledgments}

The authors acknowledge the financial support received from FAPESP (Proc. 2018/26208-0) and from National Council for Scientific and Technological Development (CNPq) [grants number 158480/2014-7 and 383876/2015-0]. The authors also acknowledge the referees, for their fruitful comments. In special, the authors acknowledge Prof. David Peters for the encouragement and for sharing with us his distinguished expertise about the Generalized Dynamic Wake Model.

\section{REFERENCES}

[1] D. Andrade. "Application of finite-state inflow to flap-lag-torsion damping in hover". Ph.D. thesis, Georgia Institute of Technology, Georgia Institute of Technology, Georgia (1992).

[2] Z. Fei. "A Rigorous solution for finite-state inflow throughout the flowfield". Ph.D. thesis, Georgia Institute of Technology, Washington University in St. Louis (2013).

[3] C. Garcia-Duffy, M.C. Hsieh \& D.A. Peters. A Complete, Nonlinear Induced Flow Theory For Rotors In Incompressible Flow. In "11th Pan-American Congress of Applied Mechanics". Foz do Iguaçu, PR, Brazil (2009).

[4] C. He. "Development and application of a generalized dynamic wake theory for lifting rotors". Ph.D. thesis, Georgia Institute of Technology, Georgia Institute of Technology, Georgia (1989).

[5] D.H. Hodges \& G.A. Pierce. "Introduction to Structural Dynamics and Aeroelasticity". Cambridge Aerospace Series. Cambridge University Press, 2 ed. (2011). 
[6] J. Morillo \& D.A. Peters. Velocity Field above a Rotor Disk by a New Dynamic Inflow Model. Journal of Aircraft, 39(5) (2002), 731-738.

[7] D.A. Peters \& W. Cao. Off-Rotor Induced Flow by a Finite-State Wake Model. In "37th AIAA SDM Conference". Salt Lake City (1996).

[8] D.A. Peters, C.J. He \& A.A. Su. Closed-form, finite-state model for the unsteady aerodynamics of rotors. Computational Mechanics, (1988).

[9] D.A. Peters, A. Hsieh \& C. Garcia-Duffy. A Complete Finite-State Inflow Theory from the Potential Flow Equations. In "Proceedings of the 3rd International Basic Research Conference on Rotorcraft Technology". Nanjing, China (2009).

[10] D.M. Pitt \& D.A. Peters. Theoretical Prediction of Dynamic-Inflow Derivatives. Vertica, 5(1) (1981), 21-34.

[11] X. Shang, D.H. Hodges \& D.A. Peters. Aeroelastic stability of composite hingeless rotors in hover with finite-state unsteady aerodynamics. Journal of the American Helicopter Society, 44(3) (1999), 206-221.

[12] Y.R. Wang. "The Effect of wake dynamics on rotor eigenvalues in forward flight". Ph.D. thesis, Georgia Institute of Technology, Georgia Institute of Technology, Georgia (1992). 